\title{
O FARMACÊUTICO NO PROGRAMA DE RESIDÊNCIA MULTIPROFISSIONAL EM SAÚDE DA UNIVERSIDADE FEDERAL DO AMAZONAS
}

\author{
Paula Chagas de Farias Rocha ${ }^{1}$ \\ Cinthya Iamille Frithz Brandão de Oliveira²
}

\begin{abstract}
RESUMO: Este relato tem como objetivo descrever as atividades realizadas pela farmacêutica residente no programa de Residência Multiprofissional em Saúde do Hospital Universitário Getúlio Vargas da Universidade Federal do Amazonas. Neste programa o farmacêutico desenvolve atividades clínicas e põe em prática a atenção farmacêutica em conjunto com uma equipe multiprofissional. A farmacêutica residente atuou na Unidade de Terapia Intensiva, onde realizou, principalmente, atividades de acompanhamento farmacoterapêutico e educação continuada com a equipe de saúde, e na Clínica Neurocirúrgica, onde a principal atividade desenvolvida foi atenção farmacêutica no preparo para a alta de pacientes com sequelas neurológicas. As dificuldades encontradas inicialmente deveram-se ao fato de que a atuação clínica do farmacêutico no Amazonas não é difundida. A atuação interdisciplinar enriqueceu a prática farmacêutica já que a abordagem passou a ser voltada ao paciente e não ao medicamento. Deficiências na formação do farmacêutico na graduação tais como: ausência de atividades interdisciplinares e formação clínica incipiente foram dificuldades superadas durante o curso. A Residência Multiprofissional possibilitou o aprendizado da prática clínica sob a supervisão de profissionais experientes neste campo suprindo a lacuna deixada na graduação.
\end{abstract}

PALAVRAS-CHAVE: Residência multiprofissional. Farmacêutico clínico. Relato de experiência.

\section{The pharmacist in multiprofessional residential program health at Federal University of Amazonas}

\begin{abstract}
This report aims to describe the activities of the pharmaceutical resident in Residence Program of Health Multiprofessional Hospital Universitário Getúlio Vargas, Federal University of Amazonas. This program develops the pharmacist clinical activities and practices pharmaceutical care in association with a multidisciplinary team. The pharmaceutical resident worked in the Intensive Care Unit, where he conducted mainly pharmacotherapeutic followup activities and continuing education with the healthcare team, and the Neurosurgical Clinic where the main activity was pharmaceutical care in preparation for discharge of patients with neurological after-effect. Initially, issues were encountered because the clinical performance of the pharmacist in Amazonas is not widespread. The interdisciplinary approach has enriched the pharmaceutical practice as the approach has been focused on the patient and not the medicine. Deficiencies in undergraduate training of pharmacists such as: lack of interdisciplinary activities and clinical training incipient difficulties were overcome during the course. The Multidisciplinary

Especialista em Análises Clínicas e Gestão Laboratorial pelas Faculdades Integradas Maria Thereza, farmacêutica residente do Programa de Residência Multiprofissional em Saúde do Hospital Universitário Getúlio Vargas da Universidade Federal do Amazonas (paulacfrocha@ yahoo.com.br).

${ }^{2}$ Doutora em Farmacologia pela Universidade Federal do Ceará, professora assistente na Universidade Federal do Amazonas (iamile@yahoo. com.br).


Residency enabled the learning of clinical practice under the supervision of experienced professionals in this field supplying the gap left at graduation.

KEYWORDS: Multidisciplinary Residency. Clinical pharmacist. Living report.

\section{INTRODUÇÃO}

Os medicamentos são tecnologias essenciais para o tratamento de pacientes enfermos por sua capacidade resolutiva, mas, ao mesmo tempo possui riscos inerentes à sua utilização. Quando usado de maneira inadequada, podem causar um impacto negativo ao usuário, acarretando problemas de ordem tanto clínica quanto econômica. Os efeitos indesejados provocados por medicamentos têm sido estudados largamente nos últimos anos. Além disso, tais efeitos podem prejudicar a efetividade das terapias medicamentosas e o objetivo terapêutico não é alcançado. Diversas são as causas para o insucesso. Elas podem estar relacionadas aos pacientes, aos profissionais de saúde, aos cuidadores ou ao próprio medicamento (BAENA et al., 2002).

Os doentes crônicos são aqueles que possuem enfermidades instaladas por um longo período de tempo, fazendo com que estes convivam com problemas de saúde, diversas internações hospitalares, limitações ou até incapacitações permanentes. Um doente crônico na família provoca alterações tanto de ordem de relacionamentos sociais quanto de ordem econômica. Os familiares passam a ser, também, seus cuidadores (FELIX; MARTINS; DYNIEWICZ, 2008).

Em alguns casos, o quadro clínico leva esses pacientes à utilização de uma grande variedade de medicamentos por muito tempo. Os doentes crônicos também podem apresentar alterações de suas funções fisiológicas normais. Diante de todo esse quadro, alguns aspectos merecem que uma atenção especial seja voltada para estes pacientes, como: ajuste de dose da droga para disfunções hepáticas e renais; monitoramento e prevenção de interações medicamentosas e reações adversas; prevenção e tratamento de infecções (KANE; WEBER; DASTS, 2003).

Dentre as doenças crônicas, cabe destacar as doenças neurológicas. Pacientes crônicos permanecem internados por grandes intervalos de tempo e muitas vezes necessitam de cuidados domiciliares. Cabe ressaltar também que algumas doenças deixam sequelas, muitas vezes irreversíveis, trazendo um impacto negativo na independência e na qualidade de vida do indivíduo (KOIZUMI, 2006).

O processo de recuperação dos pacientes com agravos neurológicos necessita de uma equipe de profissionais para trabalhar com o usuário e com sua família. A equipe multiprofissional desempenha um papel de reabilitação e também de apoio que visa proporcionar uma maior integração entre equipe de saúde, paciente e família (SMELTZER; BARÉ, 2005).

O farmacêutico, hoje em dia, vem recuperando seu papel como clínico e, com isso, buscando uma retomada na sua relação direta com o paciente. Hepler e Strand (1990) definem o Pharmaceutical Care como: a provisão responsável do tratamento farmacológico com o propósito de conseguir resultados concretos que melhorem a qualidade de vida de cada paciente. Estes resultados são 
(1) cura da doença, (2) eliminação ou redução de sintomas do paciente, (3) suspensão ou retardo do processo de doença, ou (4) prevenção de doenças ou de sintomas.

No Brasil, a atenção farmacêutica vem ser consolidada pela proposta do Consenso Brasileiro de Atenção Farmacêutica, que define a atenção farmacêutica como:

um modelo de prática farmacêutica, desenvolvida no contexto da Assistência Farmacêutica. Compreende atitudes, valores éticos, comportamentos, habilidades, compromissos e corresponsabilidades na prevenção de doenças, promoção e recuperação da saúde, de forma integrada à equipe de saúde. É a interação direta do farmacêutico com o usuário, visando uma farmacoterapia racional e a obtenção de resultados definidos e mensuráveis, voltados para a melhoria da qualidade de vida. Esta interação também deve envolver as concepções dos seus sujeitos, respeitadas as suas especificidades bio-psico-sociais, sob a ótica da integralidade das ações de saúde (OPAS, 2002, p. 16-17).

Ao praticar a atenção farmacêutica, o farmacêutico está envolvido em uma série de atividades no âmbito hospitalar (RUDIS; BRANDL, 2000; HORN; JACOBI, 2006; MONTAZERI; COOK, 1994; LEAPE et al., 1999). Dentre elas, podemos destacar, (a) análise de prescrição medicamentosa onde são avaliados dose, diluição e via de administração, posologia, incompatibilidades e interações medicamentosas; (b) participação em rounds com a equipe multiprofissional para avaliar cada paciente individualmente; (c) busca na literatura científica para elaboração e atualização de protocolos clínicos, garantindo o uso racional de medicamentos; (d) auxiliar na educação continuada, promovendo a troca de conhecimentos com toda a equipe multidisciplinar; (e) desenvolvimento da Farmacovigilância e da Tecnovigilância.

Outra atividade de grande importância é a orientação durante a preparação para alta hospitalar, visto que alguns dos principais problemas da não adesão ao tratamento são a falta de conhecimento pelo paciente e seus familiares sobre a doença, a função do medicamento utilizado e seus efeitos esperados e adversos e a correta utilização destes. Essa falta de conhecimento pode estar relacionada não só a informação insuficiente, mas também a não compreensão das orientações transmitidas pelo profissional de saúde (AMERICAN SOCIETY OF HEALTH-SYSTEM PHARMACISTS, 1997; KERZMAN; BARON-EPEL; TOREN, 2005; SILVA; SCHENKEL; MENGUE, 2000).

O despreparo do paciente ou da família sobre os cuidados que devem ser desenvolvidos no domicílio, como a dificuldade na administração de medicamentos, mudanças no estilo de vida, autocuidado com necessidades especiais decorrentes da enfermidade, entre outros, podem levar a internações subsequentes que poderiam ser evitadas (PEREIRA et al., 2007).

No Amazonas, a atuação do farmacêutico na clínica e a prática da atenção farmacêutica são praticamente insípidas, restringindo-se apenas a ações isoladas. Com o intuito de mudar este panorama o Hospital Universitário Getúlio Vargas (HUGV) da Universidade Federal do Amazonas (UFAM) contemplou vagas para farmacêuticos no seu programa de residência multiprofissional em saúde iniciado em 2010. Neste programa, o farmacêutico desenvolve atividades clínicas e põe em prática a atenção farmacêutica em conjunto com uma equipe multiprofissional. 
Diante do exposto, este trabalho tem como objetivo relatar a atuação de uma farmacêutica residente na Unidade de Terapia Intensiva (UTI) e na preparação para alta hospitalar de pacientes submetidos a neurocirurgias no HUGV durante especialização nos moldes de treinamentos em serviço que é a Residência Multiprofissional em Saúde.

\section{RELATO DE EXPERIÊNCIA}

Em 2009, por meio da Portaria Interministerial n ${ }^{\circ}$ 1077, os Ministérios da Saúde e Educação instituíram os Programas de Residência Multiprofissional em Saúde (PRMS) dos Hospitais Universitários Federais, considerados uma modalidade de pós-graduação lato sensu de ensino em serviço, com duração de dois anos. Em março de 2010, no Hospital Universitário Getúlio Vargas (HUGV) da Universidade Federal do Amazonas (UFAM), deram-se início às atividades de sua primeira turma de Residência Multiprofissional.

A residência é contemplada com sete profissões, sendo elas: farmácia, nutrição, enfermagem, fisioterapia, serviço social, psicologia e educação física. Os residentes foram distribuídos dentro das duas áreas de atuação: Saúde Funcional e Intensivismo. A área de Saúde Funcional era composta por profissionais da fisioterapia, psicologia, serviço social, enfermagem e educação física. A área de Intensivismo era composta por profissionais da farmácia, nutrição e enfermagem.

O HUGV é o centro de referência para cirurgias neurológicas no estado do Amazonas e nos demais estados da região Norte, sendo assim, a Residência Multiprofissional em Saúde tem sua atuação voltada ao cuidado integral dos pacientes submetidos à neurocirurgia.

No HUGV as principais neurocirurgias realizadas são as de tumor encefálico, aneurisma cerebral, hidrocefalia e tumor medular. Os pacientes que serão submetidos à neurocirurgia seguem, dentro da unidade hospitalar, o seguinte fluxo: admissão na unidade de internação neurocirúrgica para o preparo para a cirurgia e, posteriormente a realização da mesma, seguem para a UTI, e após alta da UTI retornam para a clínica de origem.

Para consolidar as atividades do farmacêutico no âmbito hospitalar, em 2010 o Ministério da Saúde publicou a Portaria 4283, em que aprova as diretrizes e as estratégias para organização, fortalecimento e aprimoramento das ações e serviços de farmácia (BRASIL, 2010). Esta Portaria, por meio de diretrizes, visa assegurar o acesso da população a serviços farmacêuticos de qualidade em hospitais, com intuito de lhe garantir a atenção integral à saúde, a humanização do cuidado e à efetividade da intervenção terapêutica.

Ao promover o uso seguro e racional, provendo a equipe multidisciplinar de informações consistentes que possibilitem subsidiar condutas, o farmacêutico contribui com a equipe interdisciplinar com o intuito de aprimorar o cuidado ao paciente. 


\section{Atuação da farmacêutica na Unidade de Terapia Intensiva}

A farmacêutica residente atuou, compondo, juntamente com residentes da enfermagem e da nutrição, a equipe de residentes multiprofissionais da UTI do HUGV. Os residentes trabalharam em conjunto com a equipe multiprofissional da UTI que era composta de médicos, enfermeiros, fisioterapeutas e técnicos de enfermagem. As principais atividades desempenhadas pela farmacêutica residente foram: acompanhamento farmacoterapêutico, provimento de informações sobre medicamentos e educação continuada em saúde para a equipe.

Realizar um acompanhamento farmacoterapêutico possibilitou detectar problemas relacionados a medicamentos, tais como: identificação de interações droga-droga e droga-nutriente; identificação e prevenção de ocorrências de reações adversas a medicamentos; ajuste de dose de medicamentos administrados aos pacientes com disfunções renais e hepáticas. Para a realização deste acompanhamento foi criado um instrumento para os registros dos dados e intervenções já que o seguimento farmacoterapêutico deve ocorrer de forma sistematizada e documentada. Para desenvolvimento deste acompanhamento foi utilizado a Metodologia Dáder com as devidas adaptações para o ambiente hospitalar (DÁDER; MUÑOZ; MARTINEZ, 2008).

O provimento de informação sobre medicamentos à equipe de saúde ocorria de duas maneiras: (1) sobre a forma de informes breves programados para as reuniões de passagem de plantão e (2) de acordo com a necessidade do profissional que procurava a informação. Para dispor de material de alto nível de evidência científica recorria-se às bases de dados científicas disponíveis para consulta online, tais como: PUBMED, Biblioteca Cochrane, LILACS, SCIELO, MICROMEDEX ${ }^{\circledR}$, entre outros. O material encontrado era analisado quanto a sua aplicabilidade à realidade local e transmitida ao profissional solicitante da informação.

As ações educativas desenvolvidas eram voltadas à equipe e visavam o uso racional de medicamentos. Ocorriam orientações sobre a correta armazenagem dos medicamentos e sua diluição segura, incompatibilidades entre medicamentos, utilização de equipamento próprio para medicamentos fotossensíveis, correta manipulação de medicamentos administrados via sonda de alimentação enteral, entre outros. Todas essas medidas tinham o objetivo de promover o uso seguro e eficaz dos medicamentos.

Inicialmente, foram encontradas dificuldades na atuação, pois este serviço farmacêutico não era realizado anteriormente pelo Setor de Farmácia Hospitalar do hospital. Com a aceitação gradual do trabalho desenvolvido, um elo profissional foi estabelecido com a equipe. Este elo possibilitou a evolução do cuidado com o paciente, que continua com a nova turma de farmacêuticos residentes. Com o vínculo estabelecido, a assistência integral ao paciente na terapia intensiva vem tornando-se realidade no Hospital Universitário Getúlio Vargas.

Estudos mostram que a inserção do farmacêutico no trabalho interdisciplinar na terapia intensiva só traz benefícios. A revisão de literatura realizada por Kane e colaboradores (2003) mostrou que a inserção do farmacêutico na equipe da UTI proporcionou redução de eventos como: reação adversa a medicamentos, erros de medicação, pneumonia associada à ventilação mecânica, entre outros. 


\section{Atuação da farmacêutica na clínica neurocirúrgica - a preparação para a alta hospitalar}

Segundo o plano pedagógico da instituição, o farmacêutico residente atuaria somente na unidade de terapia intensiva. Mas, ao identificar a necessidade do acompanhamento farmacoterapêutico após a alta do paciente da UTI, foi desenvolvido um trabalho ampliado dando continuidade à assistência na fase de recuperação na clínica neurocirúrgica.

Durante o acompanhamento na clínica, percebeu-se que os pacientes e seus cuidadores também precisariam de orientações para o domicílio, pois como são pacientes com afecções crônicas, suas necessidades são, em sua maioria, permanentes. Com isso, a farmacêutica e a nutricionista residente passaram a integrar a equipe de Saúde Funcional no desenvolvimento do programa que prepara o paciente para a alta hospitalar.

Esse programa baseia-se na orientação e na capacitação de cuidadores de pacientes com sequelas neurológicas crônicas para prestar assistência domiciliar de qualidade, de forma que mantenham a integridade da saúde e a boa qualidade de vida de seus entes enfermos, tornando-os mais informados em relação à patologia, seguros e hábeis para a realização dos cuidados necessários.

A primeira etapa do programa consistiu em visita e reunião interdisciplinar. Nesta reunião, as condições de saúde dos pacientes eram discutidas de maneira individualizada e planos de ação de cada profissional eram traçados para cada caso. Em seguida, após a definição do plano de atuação do paciente era agendada uma reunião com os familiares. Por meio de palestras, eram abordados temas já estabelecidos e necessários à clínica de cada paciente. Neste momento era incentivada a integração dos familiares em prol do paciente. Identificava-se com a família a necessidade de obtenção de recursos oferecidos por programas assistenciais locais, como: medicamentos de uso contínuo, órteses, nutrição enteral, equipamentos de saúde, entre outros.

Neste contexto, o papel do farmacêutico foi garantir a adesão ao tratamento medicamentoso, um dos mecanismos para a promoção do uso racional de medicamentos. Reiners e colaboradores (2008) realizaram uma revisão da literatura, identificando fatores que contribuem para a não adesão ao tratamento, dentre eles: prescrição de esquemas terapêuticos não adequados ao paciente (farmacológicos ou não), dificuldades financeiras, intolerância aos medicamentos, pouco conhecimento da doença e do tratamento, entre outros.

Para o desenvolvimento do trabalho à beira do leito, foi utilizado material impresso com quadros explicativos sobre a correta administração dos medicamentos e o passo a passo sobre a manipulação dos medicamentos, principalmente os administrados por sonda de alimentação enteral. Todo material continha mais ilustrações do que texto, uma vez que boa parte da população atendida era composta por pessoas com baixo grau de escolaridade. Durante as visitas também foram tiradas dúvidas sobre a importância do uso racional de medicamentos, as interações com alimentos e outros medicamentos. As figuras abaixo mostram alguns dos impressos utilizados na orientação aos cuidadores. 
Figuras 1 e 2 - Orientação sobre a importância do correto uso dos medicamentos.
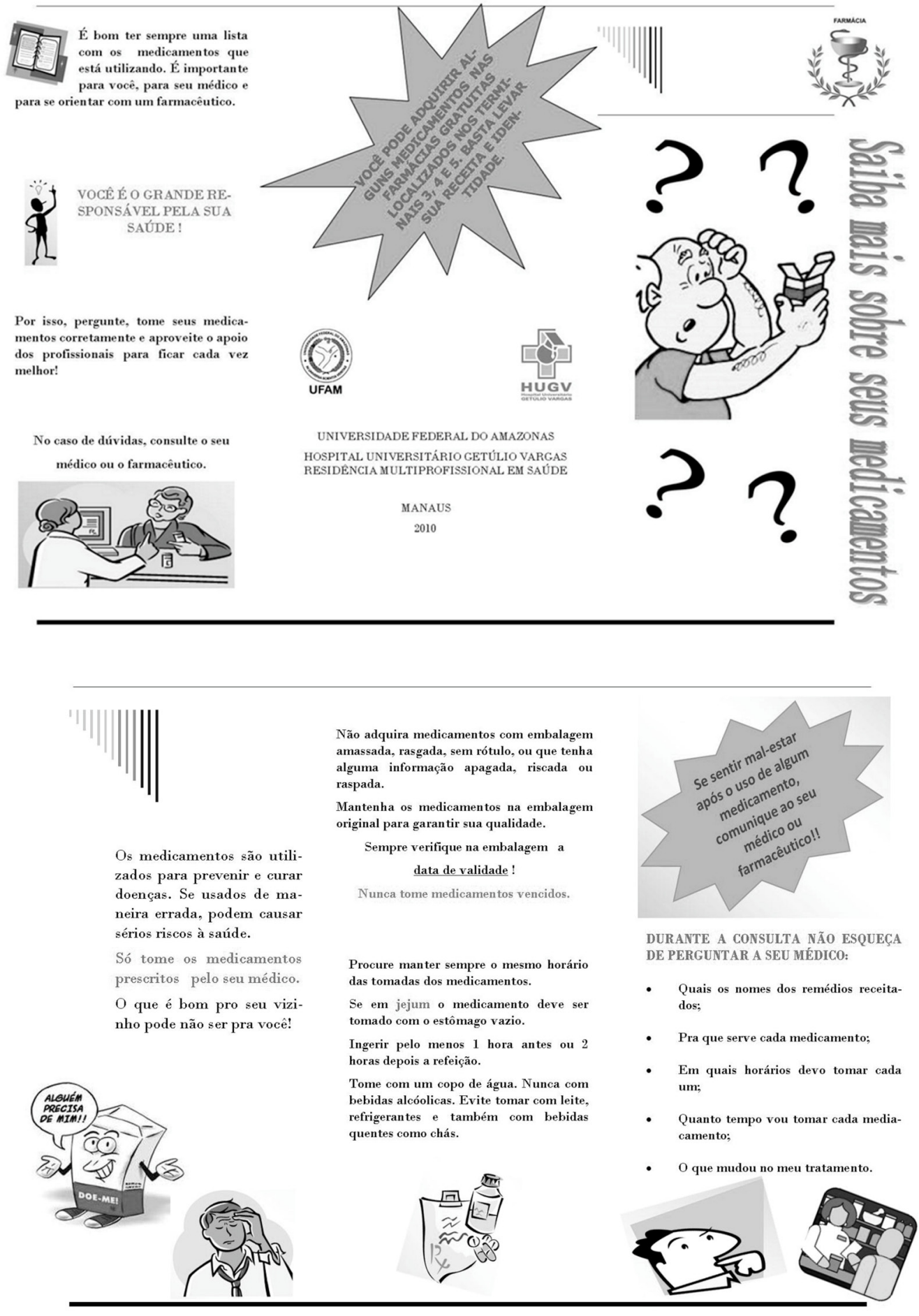

Fonte: Programa de Residência Multiprofissional em Saúde do Hospital Universitário Getúlio Vargas da Universidade Federal do Amazonas. 
Figura 3 - Tabela para orientação dos horários dos medicamentos.

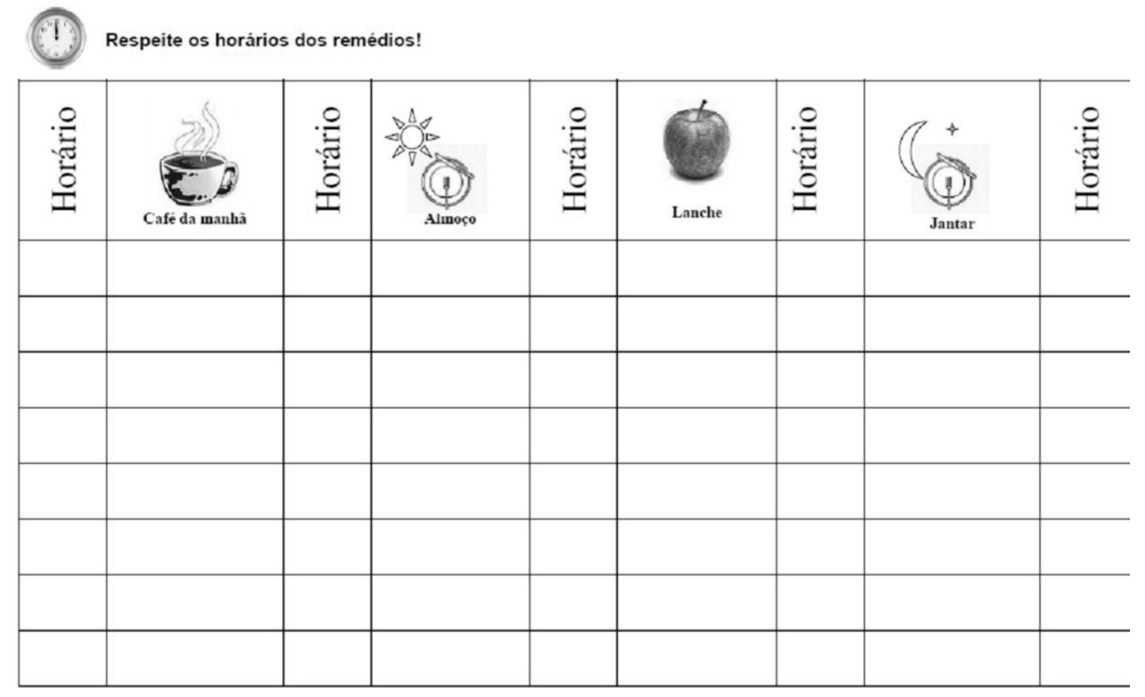

Fonte: Programa de Residência Multiprofissional em Saúde do Hospital Universitário Getúlio Vargas da Universidade Federal do Amazonas.

Figura 4 - Orientação administração medicamento via sonda.

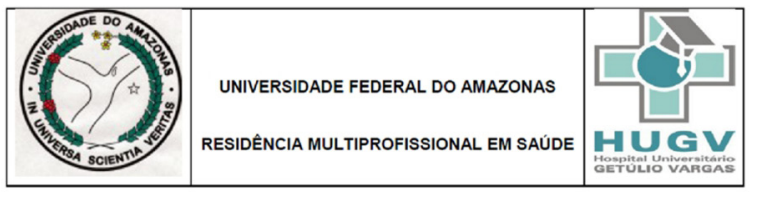

TRUTURAGÄO DE GOMPRNWNDOS

PASSO A PASSO

\begin{tabular}{|c|c|}
\hline \multicolumn{2}{|c|}{ โ Sempre lave as mãos antes de iniciar a preparação do(s) medicamento(s). } \\
\hline \multicolumn{2}{|c|}{$\begin{array}{l}23 \text { Separar, lavar e secar o material necessário. } \\
\text { tencãão: cada comprimido só deve ser retirado da sua } \\
\text { embalagemno momento de ser triturado! }\end{array}$} \\
\hline $\begin{array}{l}\text { Atenção: se ho } \\
\text { triturá-los se } \\
\text { in } \\
\text { in }\end{array}$ & $\begin{array}{l}\text { 5) Triturar bem o comprimido. } \\
\text { ver mais de um comprimido a ser administrado, } \\
\text { aradamente. Medicamentos diferentes podem } \\
\text { eragir' e até mesmo perder a ação! }\end{array}$ \\
\hline \multicolumn{2}{|c|}{$\begin{array}{l}\text { Acrescentar } 10 \mathrm{~mL} \text { de água ao triturador, lavando bem suas paredes, e mexer } \\
\text { até dissolver o comprimido triturado. }\end{array}$} \\
\hline (5) & $\begin{array}{l}\text { Antes de administrar o medicamento, } \\
\text { lavar a sonda com } 30 \mathrm{~mL} \text { de áqua. }\end{array}$ \\
\hline $\begin{array}{l}\text { (6) Administrar amistura } \\
\text { formada no triturador } \\
\text { (comprimido triurado }+10 \mathrm{~mL} \\
\text { de água) com seringa de } 20 \\
\mathrm{~mL} \text { empurrando seu êmbolo } \\
\text { vagarosamente. }\end{array}$ & $\begin{array}{l}7 \text { Após o témino da administraçãa, } \\
\text { lavar a sonda com } 30 \mathrm{~mL} \text { de água. } \\
\text { Atenção. Se houver mais de um } \\
\text { medicamento a ser administrado, lavar a } \\
\text { sonda com. } 5 \mathrm{ml} \text { de água entre uma } \\
\text { administraçãa e outra. }\end{array}$ \\
\hline \multicolumn{2}{|c|}{$\begin{array}{l}\text { OBSERVAÇÕES: } \\
\text { FECHE O SISTEMA DE ALIMETAÇÃ DA SONDA } 30 \text { MINUTOS } \\
\text { ANTES DE FAZER A MEDICAÇÃO E ABRA DE NOVO A SONDA DE } \\
\text { ALIMENTAÇÃO } 30 \text { MINUTOS DEPOIS DE FAZER A MEDICAÇÃOO. }\end{array}$} \\
\hline
\end{tabular}

Fonte: Programa de Residência Multiprofissional em Saúde do Hospital Universitário Getúlio Vargas da Universidade Federal do Amazonas. 
Sobre medicamentos, os cuidadores foram orientados a adquiri-los nas farmácias gratuitas e no programa de fornecimento de medicamentos da prefeitura de Manaus. No caso do medicamento prescrito não constante na lista da prefeitura, verificou-se com o médico a possibilidade de prescrição de medicamento de melhor custo-benefício, aliando eficácia terapêutica com menor custo. Visando também o cumprimento da terapêutica, era conversado com o médico sobre a adequação da prescrição a fim de promover um maior conforto ao paciente na administração dos medicamentos.

A inserção do farmacêutico na equipe multiprofissional, atuando de maneira interdisciplinar, proporciona ao paciente um atendimento integral. A incorporação do farmacêutico em uma equipe que une diversos saberes da saúde ajuda a garantir a melhoria da utilização dos medicamentos, com redução dos riscos de morbimortalidade e proporciona meios para que os custos relacionados à farmacoterapia sejam os menores possíveis para a sociedade (VIEIRA, 2007).

\section{CONSIDERAÇÕES FINAIS}

Oprojeto da Residência Multiprofissional busca priorizar a educação dos profissionais de saúdee impele a transformações do trabalho no setor saúde, para que o serviço e o processo de trabalho sejam espaços de apropriação de conhecimentos, atuação crítica, reflexiva, propositiva e tecnicamente competente.

As atuações interdisciplinares proporcionam a aproximação e o conhecimento dos diferentes saberes, bem como amplificam o olhar do profissional de saúde sobre os problemas de saúde pública, melhorando a comunicação entre os membros da equipe.

A experiência de atuação com outros profissionais enriqueceu a prática farmacêutica, já que o entendimento do paciente de maneira holística possibilitou uma abordagem mais assertiva, não só focando o medicamento, mas sim o indivíduo e suas particularidades. A dificuldade encontrada durante o aprendizado dessa prática foi a falta desse tipo de abordagem durante a formação profissional. A formação acadêmica enriqueceria se houvesse momentos interdisciplinares com acadêmicos da área de saúde.

Outra dificuldade encontrada foi a atuação clínica, uma vez que a formação clínica no curso de farmácia é incipiente. A especialização nos moldes de treinamento em serviço, como a residência multiprofissional, é uma excelente oportunidade para suprir a lacuna deixada na graduação, pois possibilita o aprendizado da prática clínica sob a supervisão de profissionais experientes neste campo. 


\section{REFERÊNCIAS}

AMERICAN SOCIETY OF HEALTH-SYSTEM PHARMACISTS. ASHP guidelines on pharmacist-conducted patient education and counseling. Am. J. Health-Syst. Pharm., Minneapolis, Minnesota, v. 54, p. 431-434, 1997.

BAENA, M. I. et al. Seguimiento farmacoterapéutico integral de pacientes em el sistema sanitário. Pharm. Care, Madrid, v. 4, n. 5, p. 325-332, 2002.

BRASIL. Ministério da Educação e Ministério da Saúde. Portaria Interministerial n $\mathbf{n}^{\mathbf{1}}$ 1.077, de 12 de novembro de 2009. Dispõe sobre a Residência Multiprofissional em Saúde e a Residência em Área Profissional da Saúde, e institui o Programa Nacional de Bolsas para Residências Multiprofissionais e em Área Profissional da Saúde e a Comissão Nacional de Residência Multiprofissional em Saúde. Disponível em: < http:// http://www.redeunida.org.br/noticia/ portaria-interministerial-no1077-de-12-de-novembro-de-2009>. Acesso em: 10 mar. 2012.

Ministério da Saúde. Portaria SAS n. ${ }^{\circ}$ 4283, de 31 de dezembro de 2010. Aprova as diretrizes e estratégias para organização, fortalecimento e aprimoramento das ações e serviços de farmácia no âmbito dos hospitais. Disponível em: < http:// http://www.brasilsus.com.br/ legislacoes/gm/107042-4283.html>. Acesso em: 10 mar. 2012.

DÁDER, M. J.; MUÑOZ, P. A.; MARTÍNEZ, F. M. Atenção farmacêutica: conceitos, processos e casos práticos. São Paulo: RCN Editora, 2008.

FELIX, A. P; MARTINS A. P; DYNIEWICZ, A. M. Capacitação de cuidadores de pacientes em alta hospitalar. Cogitare Enfermagem, Curitiba, v. 13, n. 1, p.124-31, jan.-mar. 2008.

HEPLER, C. D.; STRAND, L. M. Opportunities and responsabilities in pharmaceutical care. Am. J. Hosp. Pharm., Miami, v. 47, p. 533-543, mar. 1990. (Suplemento 1).

HORN, E; JACOBI, J. The critical care clinical pharmacist: evolution of an essential team member. Crit. Care Medicine, San Juan, v. 34, supl. 3, p. 46-51, mar. 2006.

KANE, S. L.; WEBER, R. J.; DASTS, J. F. The impact of critical care pharmacists on enhacing patient outcomes. Intensive Care Medicine, New York, v. 29, p. 692-698, 2003.

KERZMAN, H.; BARON-EPEL, O.; TOREN, O. What do discharged patients know about their medication? Patient Education and Counseling, Limerick, v. 56, p. 276-282, 2005.

KOIZUMI, M. S. Enfermagem em neurociência: fundamentos para a prática clínica. São Paulo: Atheneu, 2006.

LEAPE, L. L. et al. Pharmacist participation on physician rounds and adverse drug events in the intensive care unit. Jama, Maryland, v. 282, n. 3, p. 267-270, jul. 1999.

MONTAZERI, M.; COOK, D. J. Impact of a clinical pharmacist in a multidisciplinary intensive care unit. Crit Care Med., San Juan, v. 22, n. 6, p. 1044-1048, jun. 1994.

ORGANIZAÇÃO PANAMERICANA DE SAÚDE. Consenso Brasileiro de Atenção Farmacêutica: Proposta. Brasília, Organização Pan-Americana de Saúde, 2002. 
PEREIRA, A. P. S. et al. Alta hospitalar: visão de um grupo de enfermeiras. Rev. Enferm UERJ, Rio de Janeiro, v. 15, n. 1, p. 40-45, 2007.

REINERS, A. A. O. et al. Produção bibliográfica sobre adesão/não-adesão de pessoas ao tratamento de saúde. Ciênc. Saúde Coletiva, Rio de Janeiro, v.13, Suppl. 2, p. 2299-2306, dez. 2008 .

RUDIS, M. I.; BRANDL, K. M. Position paper on critical care pharmacy services. Society of Critical Care Medicine and American College of Clinical Pharmacy Task Force on Critical Care Pharmacy Services. Crit Care Med., San Juan, v. 28, n. 11, p. 3746-3750, nov. 2000.

SILVA, T.; SCHENKEL, E. P.; MENGUE, S. S. Nível de informação a respeito de medicamentos prescritos a pacientes ambulatoriais de hospital universitário. Cad. Saúde Pública, Rio de Janeiro, v. 16, n. 2, p. 449-455, 2000.

SMELTZER, S. C.; BARÉ, B. G. Tratado de enfermagem médico-cirúrgica. 9 ed. Rio de Janeiro: Guanabara Koogan, 2005.

VIEIRA, F. S. Possibilidades de contribuição do farmacêutico para a promoção da saúde. Ciência \& Saúde Coletiva, Rio de Janeiro, v. 12, n. 1, p. 213-220, 2007. 